\title{
Lumen
}

Selected Proceedings from the Canadian Society for Eighteenth-Century Studies

\section{Échanger ou (se) donner le change : la correspondance d'Émilie du Châtelet et de Jean-François de Saint-Lambert}

\section{Michèle Bocquillon}

Volume 23, 2004

URI : https://id.erudit.org/iderudit/1012191ar

DOI : https://doi.org/10.7202/1012191ar

Aller au sommaire du numéro

Éditeur(s)

Canadian Society for Eighteenth-Century Studies / Société canadienne d'étude du dix-huitième siècle

ISSN

1209-3696 (imprimé)

1927-8284 (numérique)

Découvrir la revue

Citer cet article

Bocquillon, M. (2004). Échanger ou (se) donner le change : la correspondance d'Émilie du Châtelet et de Jean-François de Saint-Lambert. Lumen, 23, 151-163. https://doi.org/10.7202/1012191ar

Copyright (c) Canadian Society for Eighteenth-Century Studies / Sociéte canadienne d'étude du dix-huitième siècle, 2004
Ce document est protégé par la loi sur le droit d'auteur. L'utilisation des services d'Érudit (y compris la reproduction) est assujettie à sa politique d'utilisation que vous pouvez consulter en ligne.

https://apropos.erudit.org/fr/usagers/politique-dutilisation/ 


\title{
7. Échanger ou (se) donner le change : la correspondance d'Émilie du Châtelet et de Jean-François de Saint-Lambert
}

\author{
[...] quand vous m'aimez comme vous $m^{\prime}$ aimez par cette poste ${ }^{1}[\ldots]$ \\ (p. 232)
}

\section{Le commerce épistolaire}

Dans un article récent ${ }^{2}$, Roland Bonnel commente la correspondance scientifique d'Émilie du Châtelet qui a, dit-il, pour «toile de fond la lutte entre les tourbillons de Descartes et l'attraction newtonienne» : mouvement et théorie de la circulation s'il en est. Émilie entretient à cette époque, c'est-à-dire de février 1738 à juin 1739, une correspondance suivie avec Maupertuis. Je ne m'arrêterai pourtant pas à cette correspondance scientifique mais à celle, plus intime, qu'elle échangera dix ans plus tard avec Saint-Lambert — du printemps 1748 à l'été 1749, soit une période de dix-huit mois : la mort d'Émilie y mettra un terme. Dans cette correspondance, il est question, cette fois, de fatale attraction et des tourbillons qui en résultent. Émilie rencontre Jean-François à la cour du roi Stanislas ${ }^{3}$. En effet, celui-ci invite Madame du Châtelet et Voltaire à séjourner à Lunéville en février 1748. Ces derniers sont installés à Cirey depuis 1735 . S'ils vivent toujours ensemble, leur relation s'est trans-

1 Émilie du Châtelet, Lettres d'amour au marquis de Saint-Lambert, textes présentés par Anne Soprani, Paris, Éditions Paris-Méditerranée, coll. "Cachet Volant», 1997. Toutes les citations renvoient à cette édition.

2 Femmes en toutes lettres. Les Épistolières du XVIII siècle, textes réunis et présentés par Marie-France Silver et Marie-Laure Girou Swiderski, SVEC 2000 : 4, Oxford, Voltaire Foundation, p. 84.

3 Roi de Pologne chassé de son trône, Stanislas Leszczynski reçut en 1738 la jouissance à vie du duché de Lorraine. 
formée, au cours des années, au grand dam d'Émilie, de l'amour passion «au sentiment paisible de l'amitié ${ }^{4}$ ». Dans son Discours sur le bonheur ${ }^{5}$, Émilie relate la souffrance que lui a causée la transformation qui s'est opérée, malgré elle, et qui la laisse désemparée. En février 1748, au moment de sa rencontre avec Saint-Lambert, il y a donc une place à prendre dans son cœur.

En dix-huit mois de relation, Émilie et Jean-François se sont vus la moitié du temps, étant donné qu'Émilie circule entre Paris, Cirey et Lunéville. Certes, leur correspondance ne repose pas uniquement sur les absences prolongées d'Émilie - elle lui écrit tous les jours, que ce soit un billet ou un poulet pendant ses séjours à Lunéville, ou des «volumes» (p. 59) quand ils sont physiquement séparés. Quant à Saint-Lambert, il lui écrit moins fréquemment et bien moins longuement, si l'on en croit Émilie.

Le commerce de lettres nous renvoie au registre de l'économie ${ }^{6}$. En effet, cette activité repose sur un échange et pour qu'il y ait échange en économie, un certain équilibre dans la démarche s'avère souhaitable. Une circularité implicite s'impose donc pour que l'entreprise soit viable, instituant tacitement une sorte de pacte épistolaire. Curieusement, on nomme cet échange une correspondance. Si l'on s'arrête un tant soit peu sur ce que sous-(en)tend ce mot, on se doit de relever que, nonobstant la fonction de relation que le préfixe cum rend indéniable, cette dernière est rendue complexe par l'idée, suggérée par le terme correspondre, d'un rapport de conformité, d'une certaine mêmeté, d'une symétrie et d'une identité dans la relation à l'autre : correspondre avec l'autre est aussi une tentative de correspondre avec / à soi. Qu'en est-il de l'échange qui s'instaure entre Émilie du Châtelet et Jean-François de Saint-Lambert? S'agit-il réellement d'échanger ou bien de donner le change, voire se donner

4 Cité par Soprani, Lettres, p. 14.

5 Émilie du Châtelet, Discours sur le bonheur, Robert Mauzi (édit.), Paris, Les Belles Lettres, 1961.

6 «Pesée des lignes et des pages, reconnaissance de dettes, valeur relative, comptes à arranger ou à régler, quittance : les lettres forment, au sens commercial du terme, un grand livre. Tout y est consigné : le nombre et la longueur des lettres. Leur ordre d'expédition et de réception, le poids de chacune pour chacun, la comparaison des investissements respectifs, les échéances et les retards, l'identité des messagers et des lecteurs, etc. Le balancement épistolaire n'est pas que le mouvement pendulaire dans lequel la lettre de l'un entraînerait la réponse de l'autre, et vice versa ; il s'agit aussi de faire la balance des comptes.» Benoit Melançon, Diderot épistolier. Contribution à une poétique de la lettre familière au XVIII siècle, Montréal, FIDES, 1996, p. 163-164. On retrouve ce décompte précis dans la correspondance de Madame du Châtelet. 
le change? Ce que nous pouvons appréhender du discours de Saint-Lambert passe par le filtre d'Émilie, c'est-à-dire par ce qu'elle nous rapporte du discours de son amant (et/ou de l'analyse et du commentaire qu'elle en fait) car nous n'avons malheureusement pas à notre disposition les lettres de Saint-Lambert. Le discours rapporté révèle, d'une part la présence d'une hétérogénéité énonciative au cœur même de l'écriture, et d'autre part, le danger toujours possible d'une parole subvertie. Je me place donc ici du point de vue d'Émilie du Châtelet, en toute connaissance de cause.

\section{Échanger : mission impossible?}

\section{À Bar le Duc. Jeudi matin [25 avril 1748.]}

J'attends votre première lettre avec une impatience qu'elle ne remplira peut-être point. J'ai bien peur de l'attendre encore après l'avoir reçue. [...][Aimer]. J'attache à ce mot bien d'autres idées que vous. J'ai bien peur qu'en disant les mêmes choses, nous ne nous entendions pas. (p. 44 , je souligne)

Émilie est en route pour Paris, en passant par Cirey. Première absence prolongée depuis leur rencontre ; elle durera deux mois. D'emblée, le "J'ai bien peur» réitéré souligne l'anticipation d'une déception. Émilie est consciente de l'opacité du langage, de l'impuissance du discours amoureux à communiquer avec l'autre. Il y va en fait d'une interincompréhension, pour reprendre le linguiste Dominique Maingueneau qui, situant ce concept dans l'interdiscours, le fait appréhender comme «l'interaction entre formations discursives ${ }^{7} . » \mathrm{~L}^{\prime}$ interincompréhension a besoin des secours d'une traduction d'un type bien particulier, «puisqu'elle opère non d'une langue naturelle à une autre, mais d'une formation discursive à une autre, c'est-à-dire entre zones de la même langue ${ }^{8}$.» Les amants qui reprennent souvent dans leur lettre le discours de l'autre pour le commenter, l'analyser, ne le font «qu'en «traduisant» l'énoncé de cet autre, en l'interprétant à travers [leurs] propres catégories" ${ }^{9}$. Si ceci est vrai en général, cela devient manifeste dans le discours amoureux. En fait, l'affect brouille souvent les messages de

7 Dominique Maingueneau, Nouvelles tendances en analyse $d u$ discours, Paris, Hachette, 1987, p. 88.

8 Dominique Maingueneau, Nouvelles tendances, p. 88.

9 Dominique Maingueneau, Nouvelles tendances, p. 88. 
sorte qu'il n'est pas toujours facile d'être sur la même longueur d'onde. À cette difficulté d'ordre sémantique s'ajoute la «dialectique particulière de la lettre d'amour à la fois vide (codée) et expressive (chargée de l'envie de signifier le désir) ${ }^{10}{ }^{\prime}$. Autrement dit, la lettre d'amour ne dit rien si ce n'est le désir de l'autre dont on attend la réponse, c'est-à-dire la réciprocité des sentiments.

[Paris.] Ce mercredi 19 [juin 1748.]

Vous êtes si économe de vos lettres que je crains que vous ne cessiez de m'écrire, peur d'écrire une lettre de trop. (p. 89)

En termes de réciprocité, il y a d'emblée un déséquilibre ; en effet, alors que Saint-Lambert est "économe», Émilie dépense sans compter. Elle l'inonde ainsi littéralement de sa prose passionnée, visant fréquemment à le culpabiliser : stratégie qui reste cependant inopérante. Elle demande souvent réparation d'un mot, d'un geste ; elle réclame, par exemple, l'envoi du portrait miniature de Saint-Lambert (destiné à orner sa bague) qu'elle attendra pendant près d'un an :

[Plombières.] Dimanche [25 août 1748] à 2 heures,

Vous n'avez qu'à m'aimer et m'écrire des lettres un peu plus longues, songez à réparer celle du 20 (p. 60, je souligne). [...] Si vous m'aimez, voyez Girardet. Voilà la grandeur de ma bague. Je sens combien cela me manque ici. Je le veux, et je l'exige et je le mérite. (p. 120, je souligne)

Le portrait doit pallier le manque de l'autre et le manquement des lettres. Le ton affirmatif et intransigeant de la dernière phrase est renforcé par l'apparence matérielle de celle-ci ${ }^{11}$; elle est écrite en bas de page, dans la bordure dentelée du papier, toutes ses lettres sont bien détachées les unes des autres - signe visible, par ce détachement calculé, de la tentative d'imposer sa volonté, de se faire bien comprendre (comme lorsque l'on articule et détache bien les mots en parlant pour attirer l'attention de son auditeur). Un détachement des lettres qui révèle paradoxalement l'attachement indéfectible à l'aimé dont elle revendique la présence, quitte à se satisfaire pour l'instant d'une représentation le portrait miniature.

10 Roland Barthes, Fragments d'un discours amoureux, Paris, Seuil, 1977, p. 187.

11 J'ai compulsé les lettres d'Émilie du Châtelet à la Pierpont Morgan Library de New York. 
Émilie ressasse son bonheur «empoisonné» (mot qui revient souvent sous sa plume), car elle aime avec excès et lui, «par accès». Cette défaillance amoureuse de l'amant se traduit par un relâchement dans sa pratique épistolaire très inégale, tant au niveau de sa fréquence que de son contenu. De fait, ses accès ne peuvent conduire qu'à l'imprévisible, alors que l'excès d'Émilie exige une correspondance régulière, voire rituelle, puisqu'il s'agit de ne pas manquer une seule poste. Il faut, malheureusement, tenir compte des aléas de la poste, d'une circulation postale non fiable à l'époque, attendu que la distribution du courrier est bien souvent perturbée par les imprévus et les hasards de la condensation (recevoir plusieurs lettres le même jour), les inversions chronologiques, les retards, la perte même - l'objet irrémédiablement perdu n'arrivant jamais à destination ${ }^{12}$. La perte, voire le détournement de lettres par autrui ${ }^{13}$, devient rapidement un leitmotiv angoissant:

\section{À Paris. Ce 10 juin [1748.]}

Il arrive certainement quelque chose de singulier aux lettres que je vous écris. Je n'ai pas passé une seule poste sans vous écrire (j'ai du moins cet avantage-là sur vous), et vous me parlez de deux postes sans avoir eu de lettres, cela est incroyable! (p. 79)

La réception entravée l'incite à numéroter ses lettres :

À Versailles. Lundi matin [10 février 1749.]

Numérotez vos lettres comme je fais les miennes; du moins, si on les ouvre, on ne pourra les supprimer. (p. 155)

Malgré la violation toujours possible, la numérotation devient garantie de survie, elle témoigne de l'être de la lettre qu'on ne peut dès lors faire disparaître. Mais retard et perte n'en demeurent pas moins une réalité :

12 Cette «dramatisation» est renforcée par «l'horloge épistolaire» : «Tout décalage est source de jeux sur les temps : à un degré élémentaire de conscience épistolaire, tout auteur de lettre sait que le présent de l'écriture correspond au futur de la réception, tout récepteur sait également que le présent de la réception renvoie au passé de l'expédition. Un ensemble de données constitue ce qu'il conviendrait d'appeler la «temporalité épistolaire». La simultanéité du contact n'est jamais réalisée que sous la forme d'un simulacre, d'un rêve ou d'un projet.» (Geneviève Haroche-Bouzinac, L'Épistolaire, Paris, Hachette, 1995, p. 77). Question que je n'envisage pas dans cet article.

13 Madame du Châtelet, jalouse de son amie Madame de Boufflers, la soupçonne de détourner le courrier. 
[Paris.] [23 février 1749.]

Mais comment, quand vous recevez le 16 ma 5e lettre qui doit être du 7 , ne voyez-vous pas clairement qu' on retarde vos lettres? Vous auriez dû la recevoir le 10, et à Lunéville, au moins la poste d'ensuite ; vous m'avez répondu le 15 à des lettres du 11, donc celle du 7 a été retardée. Il vous en manque encore une du 9. (p. 161)

À Trianon. Ce 29 [avril 1749 14 .]

Il se perd des lettres, j'en suis sûre. (p. 209)

L'absence de la lettre, qu'elle soit réelle (décevant l'attente par sa nonarrivée) ou même parfois imaginaire et/ou symbolique (ne répondant pas aux critères formels et sémantiques établis par Émilie - «J'ai bien peur de l'attendre après l'avoir reçue», p. 44), la plonge dans l'angoisse, c'est-à-dire «la confrontation du sujet à l'absence d'objet ${ }^{15}$.»

[Paris.] Dimanche 11 mai [1749.]

Point de lettres de vous aujourd'hui, voilà qui est affreux. Ce n'est pas pour me rendre ma confiance et la tranquillité d'esprit nécessaire à la vie que je mène. Imaginez, si vous pouvez, ce que c'est que d'être du jeudi au dimanche à attendre une lettre et que cette lettre n'arrive point. (p. 213)

\section{Se donner le change ou l'amaurose de l'amoureuse}

Émilie doit non seulement s'accommoder des perturbations postales, $\mathrm{du}$ retrait de la lettre de la circulation, qui bouleversent l'échange, devenu fatalement lacunaire, mais aussi du dialogue de sourds qui s'installe peu à peu puisque, si Saint-Lambert lui écrit, il ne lui répond pas, compromettant ainsi tout échange possible. En effet, échanger ne se limite pas au «donnant-donnant» matériel, mais exige une réponse aux demandes spécifiques du correspondant. Émilie en est ainsi rapidement réduite aux monologues :

[Versailles.] Lundi [21 avril 1749.]

Vous ne répondez plus à mes lettres, elles sont toujours du moins le double des vôtres, et vous laissez beaucoup de choses sans réponse (p. 202, je souligne)

[Paris.] Dimanche 11 mai [1749.]

[...] depuis longtemps vous m'écrivez sans me répondre (p. 214)

14 L'édition Soprani donne la date du 27, mais il s'agit en fait du 29.

15 Jacques Lacan, Le Séminaire IV, «La Relation d'objet», Paris, Seuil, 1994, p. 345. 
[Paris.] [Mai 1749.]

On n'aime point quand on élude, qu'on écrit peu, qu'on ne veut pas répondre, de crainte de se brouiller encore. C'est en répondant qu'on ne se brouille point. (p. 225)

La non-réponse chronique ${ }^{16}$ de Saint-Lambert dénote sa disposition à $1^{\prime}$ irresponsabilité ${ }^{17}$. Émilie ne cesse de le lui reprocher ${ }^{18}$, surtout durant son séjour de cinq mois à Paris en 1749 où, enceinte, elle travaille sans répit afin d'achever, avant son accouchement, sa traduction et son commentaire des Principes mathématiques de la philosophie naturelle de $M$. Newton ${ }^{19}$.

Émilie est consciente du leurre dans lequel elle est engagée ; en amoureuse qui se respecte, elle ne peut se contenter d'une vision superficielle des choses. En effet, comme le souligne Barthes :

L'amoureux, c'est le sémiologue à l'état pur ! Il passe son temps à lire les signes. Il ne fait que ça : des signes de bonheur, des signes de malheur. Sur le visage de l'autre, dans ses conduites. Il est véritablement en proie aux signes. [...] Il voit clair. Mais le résultat est souvent le même que s'il était aveugle ${ }^{20}$.

Émilie voit tout en profondeur, elle lit entre les (rares) lignes de JeanFrançois et pourtant sa lecture semble biaisée par une volonté de ne pas

16 Faute d'avoir à notre disposition les lettres de Saint-Lambert, on ne peut que supposer qu'il ne répond pas, entre autres, aux questions concernant son attachement à Madame de Boufflers. L'inconstance présumée de Jean-François d'un côté, la jalousie d'Émilie de l'autre, parasitent ainsi l'échange.

17 Selon Emmanuel Levinas, Eros incarne «la séduction de l'irresponsabilité», autrement dit l'attrait de la non-réponse, donc fatalement de la non-communication; on se détourne de l'autre après consommation. Totalité et infini : Essai sur l'extériorité, La Haye, Nijhoff, 1961, p. 59.

On prend et on quitte, on fait circuler : Philip Stewart souligne cette morale de l'irresponsabilité bien adaptée au XVIII ${ }^{\mathrm{e}}$ siècle dans Le Masque et la parole, Paris, Librairie José Corti, 1973.

Émilie craint constamment d'être abandonnée par Saint-Lambert : «[...], si vous m'abandonnez. Vous me présentez trop souvent cette idée pour que je la perde ; $[\ldots]$ » (p. 83).

18 «C'est ce manque d'empressement de Saint-Lambert, sa façon négligée de se laisser aimer, son non-engagement qui dominent dans les lettres d'une Émilie, à l'opposé, emportée et exigeante.» (Soprani, Lettres, p.18).

19 Préfacé et publié par Voltaire, Paris, Desaint et Saillant, 1759.

20 Roland Barthes, Le Grain de la voix, Paris, Seuil, 1981, p. 282. 
savoir, ce que je nomme l'amaurose de l'amoureuse ${ }^{21}$ (amaurôsis : obscurcissement de la vision) qui perturbe inévitablement la communication, puisqu'elle repose sur le simulacre, la simulation et l'(auto)dissimulation. Elle tente parfois même de voir trop loin, déformant ainsi, par sa traduction du discours de l'autre, la représentation des choses : une anamorphose qui étaye et renforce l'amaurose. La «géomètre ${ }^{22}$ » en vient même à renoncer à son esprit de géométrie :

[Paris.] Vendredi soir [28 mars 1749.]

Non, je douterai plutôt de mon existence, je croirai plutôt que 2 et 2 font 5 , que de douter un moment que vous ayez la plus belle âme du monde. Trompez-moi toujours, mais ne m'ôtez pas cette idée qui m'est plus chère que la vie (p. 173, je souligne)

Sa volonté de ne pas savoir va jusqu'au déni de la réalité, elle en arrive ainsi à se donner le change. Elle ne peut renoncer à sa fixation, persiste et signe dans sa volonté de faire advenir l'amant idéal, malgré les indices évidents de son insuffisance. D'une lettre à l'autre, on découvre dans l'expression d'Émilie le renversement en son contraire - selon la dualité du principe plaisir-déplaisir — de «l'état de [son] cœur» (p. 80) : quelques lettres séparent «Mon dieu, que je suis heureuse !» (p. 44) de «Mon dieu, que je suis malheureuse, [...]!» (p. 49) - structure syntaxique identique qui rend d'autant plus troublante l'antinomie sémantique. Ce retournement est en soi symptomatique de la dynamique relationnelle. Émilie est le plus souvent malheureuse dans ses moments de lucidité où elle tente de voir Saint-Lambert dans toute son humanité, si décevante soit-elle ; elle est heureuse quand elle s'adonne à la divination de l'aimé. L'amant selon son cœur correspond alors à son fantasme. Comme le souligne Brigitte Diaz : «La communication épistolaire a ceci d'idéal qu'on s'y adresse à un autre imaginaire, non pas tel qu'en lui-même, mais tel qu'on le rêve ${ }^{23}$ "). La lettre circule ainsi entre réel et fiction : réalité de l'interaction épistolaire certes, mais fiction, de par la création du partenaire idéal le destinataire est un autre.

[Choisy.] Ce lundi 27 [mai 1748] à deux heures après minuit.

Vous ne pouvez avoir tort. Je vous adore, je ne sais, je ne sens, je ne vois que cela!

21 L'amaurose est une perte soudaine de la vue.

22 "Quoique je sois peut-être plus géomètre que vous, je ne suis pas si compassée» (p. 88.)

23 Brigitte Diaz, L'Épistolaire ou la pensée nomade, Paris, PUF, 2002, p. 59. 
(p. 64, je souligne)

Elle s'en remet ainsi entièrement à l'être aimé. Elle se complaît dans l'aveuglement puisqu'elle ne voit qu'un objet d'adoration, un objet auratique ${ }^{24}$.

À Versailles. Lundi matin [10 février 1749.]

Croyez, mon cher amour, croyez, bonheur de ma vie, que jamais mon cœur n'aura avec vous un moment de distraction ni de négligence. Votre cœur, votre amour, la chaleur et la sensibilité de votre âme sont pour moi la pierre précieuse pour la conservation de laquelle je donnerais tout ce que j'ai et tout ce que je suis (p. 155-156, je souligne)

[Paris.] Dimanche [8 juin 1749.]

Votre cœur charmant, tel que vous me le montrez dans vos deux lettres que je viens de recevoir à la fois, est pour moi la pierre précieuse de l'Évangile. Je veux tout sacrifier pour en jouir, pour le conserver (p. 231, je souligne)

Dans les moments d'euphorie amoureuse suscitée par la réponse de l'amant (cette fois conforme à l'attente) et/ou par un service postal qui se surpasse en délivrant deux missives à la fois, Émilie s'adonne à la divination de l'humain et métamorphose l'aimé en trésor, en "pierre précieuse». Autrement dit, elle le transforme en capital : richesse dont la conservation exige paradoxalement l'appauvrissement de celle qui en a la jouissance (dans les deux sens du terme). En fait, elle est prête à tout sacrifier à cette jouissance pourtant jamais réellement appréhendée, toujours différée et en dernier ressort impossible, sa constance ne pouvant se satisfaire à terme de la versatilité, des «disparates» de Saint-Lambert. Seule l'écriture lui permet de rester à la surface et de faire face, malgré l'impression de "passe[r] [s]a vie à faire avec [lui] des monologues» (p. 165). L'échange se fait donc insensiblement avec elle-même.

Entre les amants, il s'agit donc bien souvent - si l'on en croit Émilied'un conflit entre le trop et le peu, d'un déséquilibre affectif fondamental qui mène à la faillite, la balance des comptes ne pouvant être que déficitaire pour l'amoureuse.

24 L'aura de l'objet est caractérisée par l'unicité, la proximité et la distance de ce dernier (à la fois présent et absent) qui le rend en quelque sorte inapprochable : «Qu'est-ce proprement que l'aura ? Une trame singulière d'espace et de temps : unique apparition d'un lointain, si proche soit-il.» Walter Benjamin, «Petite histoire de la photographie», CEuvres II. Poésie et Révolution, Paris, Denoël, 1971, p. 27. Cette définition sera reprise dans "L'CEuvre d'art à l'ère de sa reproductibilité technique», p. 178. 
Comme dans un système de vases communicants, dans les périodes de longue absence - particulièrement les deux périodes parisiennes la lettre enfle, souffle, et le corps d'Émilie s'essouffle, s'étiole, l'une s'élargit en étalant les états d'âme, l'autre rétrécit sous l'effet de luttes intestines - lutte contre cette fatale attraction dont les tourbillons l'entraînent irrésistiblement. Ne dit-elle pas ailleurs, dans une lettre scientifique à Algarotti dans laquelle elle joue sur les différents sens du mot tourbillon : «Je connais le tourbillon et combien il est difficile de n'en pas être emporté25 " ? Si Émilie renonce au tourbillon de la vie parisienne pour se consacrer exclusivement à «son Newton», dans l'espoir de rejoindre Saint-Lambert au plus vite, elle se laissera cependant emporter complètement par les tourbillons tumultueux de ses amours.

Cette pratique épistolaire combative - ce jeu d'escrime avec l'autre mais surtout avec elle-même ("ma tête est un chaos de contradictions», p. 202) qui permet selon le coup donné ou reçu de passer du malheur au bonheur et vice versa - finira par miner ses forces vitales. Cette correspondance (si l'on peut encore l'appeler ainsi, étant donné les manifestations sporadiques de Saint-Lambert) épuisante, toujours relancée, sera le chant du signe d'Émilie, puisque celle qui s'est déchaînée dans et enchaînée par l'écriture finira par renoncer : "Je finis car je ne peux plus écrire $^{26}$ » (p. 243). À la boulimie scripturale qui tenta de tisser un lien inaliénable succède le silence ou encore la paralysie ; la paralysie, telle que définie par Derrida, qui nous rappelle son étymon :

[...] la paralysie, ça ne signifie pas qu'on ne peut plus bouger ni marcher, mais en grec, s'il te plaît, qu'il n'y a plus de lien, que toute liaison est dénouée (autrement dit, analysée) et qu'à cause de cela, parce que l'on est «exempté», acquitté de tout, rien ne va plus, rien ne tient plus ensemble, rien n'avance plus. Il faut du lien et du nœud pour faire un pas ${ }^{27}$.

En d'autres termes, il faut de l'imaginaire, une liaison soutenue par le fantasme, car la relation souhaitée est toujours déjà perdue. N'écrit-elle pas dans son Discours sur le bonheur : «Il faut, pour être heureux, [...] être susceptible d'illusions, car nous devons la plupart de nos plaisirs à

25 Cité par Bonnel, p. 88.

26 Émilie ne savait pas alors qu'elle ne pourrait plus réellement écrire. Elle accoucha quelques jours plus tard, le 4 septembre 1749, et mourut le 10.

27 Jacques Derrida, La Carte postale, de Socrate à Freud et au-delà, Paris, Flammarion, 1980, p. $138-139$. 
l'illusion, \& malheureux est celui qui la perd ${ }^{28}$ » ? Or Émilie l'analyste, la sémiologue inconditionnelle, à force de lire et relire les signes - selon sa propre grille de lecture - n'avance plus, elle a dénoué les liens qui la rattachent à Jean-François. L'amaurose intermittente dont elle souffre n'a fait que reporter le dénouement. Elle aurait pu écrire : «Je finis d'écrire donc j'en finis avec moi», puisque seule l'écriture lui faisait supporter la vie. L'épreuve de réalité, si longtemps différée, malgré les spasmes de lucidité au cœur de son aveuglement (consenti) — le plaisir «n'est pas sans mélange» (p. 121), confie-t-elle - finit par s'imposer. Après avoir cru à une «réparation» toujours possible, qu'elle n'a cessé de réclamer, elle abandonne la partie et s'abandonne car, pour avoir trop longtemps scotomisé (laissé dans l'ombre) l'impossibilité de cette «relation $\mathrm{d}^{\prime}$ obje $^{29}{ }^{2}$, l'ombre de l'objet a fini par la recouvrir et lui a fait perdre tout son éclat. Elle succombe alors à une détresse physiologique. Émilie qualifie elle-même son amour d'excès, de folie, de fureur, de démence — ce qui la conduit au «dégoût [d'elle]-même» car, écrit-elle : «Il m'est impossible d'exister sans vous», dit-elle (p. 125). Ce dégoût d'elle-même préfigure la mélancolie, le renoncement, le «désêtre ${ }^{30} »$. La fixation de sa libido à l'aimé contrecarre ainsi toute mobilité future.

\section{Donner le change ou la gratification}

On décèle en filigrane de l'écriture, dans les réponses à Saint-Lambert (car Madame du Châtelet lui répond, contrairement à lui) que ce dernier entretient et alimente l'amaurose $\mathrm{d}^{\prime}$ Émilie en lui donnant le change :

[Paris.] Jeudi 23 mai [1748.]

Comment pouvez-vous toujours me soupçonner ? Puis-je vous négliger un moment? Je jure que je vous écris toutes les postes. (p. 58)

29 L'amant passe, selon les états d'âme d'Émilie, de bon à mauvais objet et vice versa.

30 Autrement dit la perte de soi : «[...] l'acte du «laisser tomber» [...] signe alors le point où il n'y a plus de parole possible, plus d'adresse à l'Autre, plus que cet instant où le sujet, arrivé au bout de son «désêtre», choit et se rencontre enfin, dans sa chute même, dans ses épousailles mélancoliques avec lui-même dans la mort.» Article "Mélancolie», Dictionnaire de la Psychanalyse, Paris, Larousse, 1993, p. 159. Madame du Châtelet ne se suicide pas «Mais [...] le désamour de Saint-Lambert [...] l'entraîne dans une fatale dépression. À travers la trentaine de lettres qui subsistent pour l'année 1749 , on lit son lent cheminement vers l'abandon de toute énergie vitale.» (Soprani, Lettres, p. 24). 
En effet, comment comprendre ce renversement ? Tout en reprochant à Émilie ses trop longues missives, il se plaint d'autre part d'être négligé alors que c'est lui, si l'on en croit sa correspondante, qui se laisse désirer.

[Paris.] 5 juin 48.

Je relis votre lettre, elle m'enchante, mais je crains de ne la devoir qu'à mes reproches, et que vous n'ayez fait des efforts pour être tendre. Écrivez donc toujours de même et je ne craindrai rien. (p. 74 , je souligne)

Une fois de plus, Émilie n'est pas dupe. Une fois de plus, elle dénonce le manque de sincérité de son amant et pourtant, contre toute attente, elle en redemande. Quitte à se satisfaire pour un temps d'un semblant de tendresse, elle préfère le leurre de la présence affective à l'angoisse de son absence. Mais à terme le faire semblant ne peut s'avérer satisfaisant.

[Lunéville.] [Août 1748.]

Je vous ai reproché quelquefois ${ }^{31}$ de n'être pas galant, mais je trouve que votre lettre l'est trop, et qu'elle n'est point tendre. Je vous aime bien mieux en colère et tendre que froid et galant. (p. 104.)

Émilie en revient fatalement aux reproches : la lettre d'amour ne peut en aucun cas devenir lieu de galanterie, puisque celle-ci est incompatible avec la tendresse recherchée. À l'époque, comme le souligne Ruth Amossy : «Le parfait galant est celui qui l'est «trop» car il a recours à une langue apprise et conventionnelle qui n'exprime pas des sentiments véritable $^{32} . »$ Autrement dit, le parfait galant n'a rien à dire, il donne tout simplement le change.

Lorsque Émilie tente quelquefois de prendre ses distances (pour qu'un échange équitable s'opère enfin ?), la réaction ne se fait pas attendre :

[Paris.] Jeudi à 3 heures [avril 1749.]

Mais je ne sais pourquoi vous êtes furieux quand mes lettres sont froides, et vous ne répondez point quand elles sont tendres. (p. 183)

On pressent chez Saint-Lambert (d'après ce qu'en laissent présager les dires de Madame du Châtelet) le désir de recevoir sans contrepartie ; il

31 J'ai ajouté le mot quelquefois, qui n'est pas repris dans l'édition Soprani, mais qui est bien présent dans la lettre de Madame du Châtelet.

32 Ruth Amossy, «La Lettre d'amour, du réel au fictionnel», dans Jurgen Siess (édit.), $L a$ Lettre entre réel et fiction, Paris, SEDES, 1998, p. 82. 
attend, de la part d'Émilie, le don de soi - une dépense en pure perte, en somme. Il ne semble donc pas envisager la relation sur le mode de l'échange, mais il s'agit plutôt de cultiver l'amaurose pour être gratifié du don.

La lettre, véhicule et support du pacte épistolaire, se révèle donc inapte à honorer ce dernier ; la faillite du commerce des lettres est ainsi consommée puisqu'il n'y a pas d'échange réel, c'est-à-dire de démarche équilibrée misant sur la réciprocité et la circularité. Si l'on décèle une réelle volonté d'échanger chez Madame du Châtelet - échange qu'elle réclame souvent à corps et à cris, et qui se traduit par une densité matérielle impressionnante - Saint-Lambert, quant à lui, s'est contenté de lui donner le change. Émilie, malgré ses accès de lucidité au cœur de son aveuglement consenti, et peut-être finalement à cause d'eux, en vient à se donner le change afin de rester «susceptible d'illusions» ; les illusions demeurant pour elle constitutives de tout Discours sur le bonheur. En somme, la relation amoureuse reposerait, selon Émilie du Châtelet, sur la capacité de s'engager dans une fiction affective et d'en assumer seule le déroulement et ses effets ${ }^{33}$. Je laisserai le mot de la fin à Robert Mauzi qui rapporte et résume pertinemment cette dynamique :

Madame du Châtelet dans ses Réflexions sur le bonheur explique qu'il faut savoir «aimer pour deux» [...] [Les] imperfections [de l'amour] ne parviennent pas à volatiliser le bonheur d'aimer, ni cet autre bonheur, plus fragile sans doute et qui exige bien des compromis avec soi-même, celui de «se croire aimés ${ }^{34} »$.

\section{MICHÈLE BOCQUILLON}

The City University of New York

33 Pourtant, qui dit relation pense échange, réciprocité, circularité...

34 Robert Mauzi, L'Idée du bonheur dans la littérature et la pensée françaises au XVIII siècle, Paris, Albin Michel, 1994, p. 471. 